\title{
Validation of diabetes medication self-efficacy scale in Chinese with type 2 diabetes
}

This article was published in the following Dove Press journal:

Patient Preference and Adherence

\author{
Jiezhong Zheng' \\ Yeli Wang ${ }^{2}$ \\ Xiangrong $\mathrm{Ye}^{3}$ \\ Ling $\mathrm{XiaO}^{4}$ \\ Junting $\mathrm{Ye}^{5}$ \\ Xiaoru $\mathrm{Li}^{6}$ \\ Mingkang Zhong'
}

'Department of Pharmacy, Huashan Hospital, Fudan University, Shanghai 200040, China; ${ }^{2}$ Health Services and Systems Research, Duke-NUS Medical School, Singapore I69857, Singapore;

${ }^{3}$ Department of Endocrinology, Xiamen Municipal Hospital of

Traditional Chinese Medicine, Xiamen 361009, China; ${ }^{4}$ Department of Endocrinology, Jinshan Hospital of Fudan University, Shanghai 201508, China; ${ }^{5}$ School of Basic Medical Sciences, Fujian Medical University, Fuzhou 35000I, China; ' $D$ Department of Psychology, School of Social Development and Public Policy, Fudan University, Shanghai 200433, China

Correspondence: Mingkang Zhong Department of Pharmacy, Huashan Hospital, Fudan University, 12 Middle Wulumuqi Road, Shanghai 200040, China Tel +86 02I 52888377

Email hsmkzhong@hotmail.com

Xiaoru Li

Department of Psychology, School of Social Development and Public Policy, Fudan University, 220 Handan Road,

Shanghai 200433, China

Tel +86 02I 55664532

Email lixiaoru@fudan.edu.cn
Aims: Greater diabetes medication self-efficacy has been shown to associate with better medication adherence, which is critical for disease control for people living with type 2 diabetes (T2D). The Diabetes Medication Self-efficacy Scale (DMSS) is a 19-item instrument to measure diabetes medication self-efficacy. The aim of the current study was to translate the DMSS into Chinese and validate the Chinese version of DMSS (CDMSS) among Chinese T2D patients.

Methods: The CDMSS was translated using forward-backward method, and its validity and reliability were assessed among 257 Chinese T2D patients. Exploratory factor analysis, Cronbach's $\alpha$ and Spearman's rank correlation coefficients were used to evaluate the psychometric properties of CDMSS. Receiver operating characteristic analysis was used to assess the discrimination of CDMSS for medication adherence and glycemic control.

Results: Exploratory factor analysis has generated a one-factor structure of the 11-item version of CDMSS (CDMSS-11), which accounted for $63.1 \%$ of the variance. The Spearman's rank correlation coefficient between the original 19-item and 11-item scale was 0.96. The Cronbach's $\alpha$ for internal consistency was 0.94 , and the test-retest reliability coefficient was 0.76 . The CDMSS-11 score was significantly correlated with diabetes distress, medication adherence, as well as blood levels of fasting plasma glucose and hemoglobin A1c (both $P<0.001$ ). The area under receiver operating characteristic curve and its corresponding $95 \% \mathrm{CI}$ was $0.79(0.73-0.84)$ for medication adherence, and it was $0.65(0.57-0.72)$ for better glycemic control.

Conclusion: The CDMSS-11 is a valid and reliable measure to assess medication self-efficacy among Chinese T2D patients.

Keywords: medication adherence, medication self-efficacy, type 2 diabetes, validation study

\section{Key points for decision makers}

1. The current study was the first one to translate the Diabetes Medication Self-efficacy Scale from English into Chinese and evaluate its psychometric properties among Chinese patients with type 2 diabetes, who had the highest diabetes burden in the world but low medication adherence.

2. Our results showed that the translated instrument had good validity and high testretest reliability, and had significant correlation with medication adherence in this Chinese population. In addition, we also found good discriminatory utility of this instrument for identifying people with good medication adherence and proper glycemic control.

3. Our results suggested the potential clinical implication of the translated instrument in helping clinicians to identify diabetic patients with low medication self-efficacy, poor medication adherence and bad glycemic control in China, so that the proper interventions can be introduced to manage diabetes. 


\section{Introduction}

Diabetes is an endocrine disorder featured with increased glucose levels in the blood, and has become the leading global health emergency. ${ }^{1}$ The International Diabetes Federation estimated that a total of 425 million adults had diabetes in 2017, while China ranked first (114.4 million) among all the countries. ${ }^{1}$ Type 2 diabetes (T2D) accounts for approximately $90 \%$ of all cases of diabetes, ${ }^{2-4}$ and inappropriate management will lead to complications in various organs such as cardiovascular disease, nephropathy, retinopathy and blindness. ${ }^{1}$ Despite of effective medication therapies for T2D management, only $39.7 \%$ of T2D patients under medication treatment had adequate glycemic control. ${ }^{5}$ A known factor to less ideal glycemic control is the nonadherence of patients to prescribed medication, and a 5.4-year follow-up study among 11,272 T2D patients has shown that each $10 \%$ increment in medication adherence significantly decreased the mean levels of hemoglobin A1c (HbAlc) by $0.24 \%{ }^{6}$ However, in a systematic review of 27 studies across populations, only six studies (22.2\%) reported good medication adherence. ${ }^{7}$ In addition, a study among 163 Chinese patients from China has shown that the average medication adherence was only $52 \% .^{8}$

Self-efficacy is a central concept of social cognitive theory, and it refers to beliefs in one's capabilities to organize and execute the courses of action required to produce given attainments. ${ }^{9}$ In addition, it has been implicated as a modifiable cognitive factor for medication adherence. ${ }^{10}$ Results from a meta-analysis of 13 studies have shown a positive correlation between self-efficacy and medication adherence $(r=0.42)$, which was much higher than the correlations between other cognitive components (such as illness beliefs or treatment beliefs) and medication adherence ( $r$ ranged from 0.06 to 0.20$).{ }^{10}$ Consistently, a prospective follow-up study and a randomized clinical trial have further supported this inference by showing that self-efficacy not only reinforced medication adherence, ${ }^{11}$ but also resulted in better glycemic control. ${ }^{12}$ Therefore, it is of clinical interests to assess the medication self-efficacy, an important element of self-efficacy, in T2D patients so that individually tailored interventions can be introduced.

The Diabetes Medication Self-efficacy Scale (DMSS) is a newly-developed 19-item self-administered questionnaire with good reliability (Cronbach's $\alpha=0.86$ ) that measures T2D medication self-efficacy in English-speaking populations. ${ }^{13}$ However, the factor structure was not explored in that study, and the scale has not been validated in another population with different languages. Thus, we adapted and validated the
Chinese version of DMSS (CDMSS) in a sample of Chinese T2D patients for the first time.

\section{Methods}

\section{Study design and participants}

We validated the DMSS questionnaire within a cross-sectional study of adult T2D patients aged 18-80 years old in China. Between November 2016 and September 2017, we recruited T2D patients from outpatient clinics and inpatient wards in three hospitals in China, namely Jinshan Hospital of Fudan University in Shanghai, Xiamen Municipal Hospital of Traditional Chinese Medicine in Xiamen, and The First Affiliated Hospital of Guangzhou University of Traditional Chinese Medicine in Guangzhou. The three cities selected in the current study were all among the first-tier cities in China, and the chosen hospitals widely covered patients seeking primary care and secondary or tertiary care, and were thus representative of the general populations from economically developed cities in China. Patients were included if they: 1) could speak fluent Mandarin; 2) had received primary school education or higher; 3) had been diagnosed with T2D defined by 2016 American Diabetes Association (ADA) diagnostic criteria; ${ }^{14}$ 4) were under at least one medication treatment; and 5) had no severe diabetes complications or other diseases such as cancer and dementia.

At recruitment, after T2D patients expressed their interests in the current study, we reviewed their medical records to make sure they met the inclusion criteria 3-5, and further confirmed their T2D status by measuring their $\mathrm{HbA} 1 \mathrm{c}$ and fasting plasma glucose (FPG) levels. A total of 347 patients met the inclusion criteria and were recruited. Among them, 36 patients dropped out during the study period, 15 patients lost contact and four patients did not fill out the baseline questionnaires properly, leaving a total of 292 patients for the present analysis. Among the 292 recruited patients, the first 35 patients were invited to participate in the pilot study to develop the CDMSS, and we validated the translated DMSS in the remaining 257 patients. The flowchart of the current study is shown in Figure S1. Each participant was given a gift worthy around $20 \mathrm{RMB}(\approx 3 \mathrm{USD})$. The study protocol was approved by the Research Ethics Committee of Xiamen Municipal Hospital of Traditional Chinese Medicine, and written informed consents were provided and completed by each patient.

\section{Face and content validity Translation of DMSS}

Following the Brislin's translation model, ${ }^{15}$ the DMSS was translated into Chinese by three independent $\mathrm{PhD}$ students 
(JZ, YW, and HL). After reaching a consensus, the draft was translated back to English independently by two professors in English major (MW and YL). Discrepancies between the original and translated questionnaires were discussed, and adaptations were made to Chinese culture. Subsequently, 35 T2D patients filled out the CDMSS. We then used cognitive interviewing methodology ${ }^{16}$ to collect feedbacks about whether the questionnaire was clear and easy to understand, and made further refinements in CDMSS. The final version of CDMSS was used as the instrument to measure diabetes medication self-efficacy for the current study (Table S1).

\section{Data collection}

At recruitment, participants filled out four self-administered questionnaires to provide information on demographic characteristics, medical history, medication usage (types, frequencies and side effects), perception on T2D and related medications, diabetes medication self-efficacy and adherence, and diabetes distress. Two to four weeks later, a total of 47 participants repeated the CDMSS to examine its testretest reliability.

\section{Measures}

\section{Diabetes medication self-efficacy}

Diabetes medication self-efficacy was measured by the CDMSS, which contained 19 items that described different situations or circumstances that T2D patients may encounter in taking their medications. Each item had three choices to describe the degree of confidence ("Not at all sure", "Somewhat sure" and "Very sure"), and the corresponding score ranged from 1 to 3 . The overall score of the CDMSS was the totality of all the items, where a higher score represented better diabetes medication self-efficacy.

\section{Perception on T2D and related medications}

Perception of T2D, T2D medications and medication efficacy were assessed by the questions "How well do you know your disease (T2D)", "How well do you know your T2D medications" and "What is the efficacy of your current medication", respectively. Each patient was asked to choose from a scale ranging from $0=$ "Not at all/very bad" to $10=$ "Very well/ very good".

\section{Medication adherence}

To measure the patient medication adherence, we used the Visual Analog Scale (VAS) ${ }^{17}$ which was developed by Freyd, and has been applied widely in clinical and research settings since the 1920s. ${ }^{18-20}$ In the current study, patients were shown a $100 \mathrm{~mm}$ line with endpoints of "no adherence" at 0 and "complete adherence" at 100, and were asked to rate themselves on the scale based on the question "During the past 1 month, what percentage of time did you take all your diabetes medications as your doctor prescribed?". A score of 80 and above was defined as good medication adherence.

\section{Diabetes distress}

Diabetes distress was measured by the 15-item Chinese version of Diabetes Distress Scale (CDDS-15) developed by Ting et al and validated in a Chinese population in Hong Kong with good validity and reliability (Cronbach's $\alpha=0.90$; test-retest coefficient $=0.74) .{ }^{21} \mathrm{CDDS}-15$ contained 15 items, which assessed emotional burden, regimen- and social support-related distress, and physician-related distress of patients. ${ }^{21}$ Each item had a 6-point Likert response ranging from $1=$ "Not a problem" to $6=$ "Serious problem". The CDDS-15 used the average score of the 15 items, where a lower score represented less diabetes distress.

\section{Laboratory assay}

To measure the blood levels of FPG and HbA1c, each recruited patient was referred to the clinical laboratory located at each hospital for blood taking. The FPG levels were measured using the hexokinase method by an automatic biochemical analyzer (Roche Cobas 8000; Qingyang, Guangzhou, China), and HbA1c levels were measured using the high-performance liquid chromatography method on an automatic glycohemoglobin analyzer (Arkray ADAMS ${ }^{\mathrm{TM}}$ A1c HA-8180; Yangpu, Guangzhou, China) in the same laboratory immediately after a blood collection.

\section{Statistical analysis}

Exploratory factor analysis was performed to explore the dimensions of CDMSS using principal components analysis with both quartimax rotation and oblique rotation methods. Factors were extracted based on Eigenvalues (greater than 1). Items with loading $\geq 0.40$ within one factor were retained, while items loaded on multiple factors were deleted. In addition, internal consistency was examined by the Cronbach's $\alpha$, and test-retest reliability was examined by the intracorrelation coefficient (ICC). Spearman's rank correlation coefficients between the CDMSS, the VAS, the CDDS-15, blood levels of FPG and HbA1c, as well as the factors that were collected from self-administered questionnaires at recruitment (continuous variables) were calculated. Morevoer, Kruskal-Wallis $H$ test was used to compare the distribution of CDMSS for categorical factors from the questionnaires at recruitment. Then multivariable linear regression analysis was conducted to examine the association of these factors 
with diabetes medication self-efficacy. Furthermore, logistic regression analysis was used to compute the relation between the CDMSS and the binary HbAlc $(<$ vs $\geq$ individualized optimal treatment targets). When choosing the optimal targets for treatment, we followed the ADA's recommendation ${ }^{22}$ and chose $7 \%$ as a reasonable target for most nonpregnant adults $(n=230)$, and $8 \%$ as a less stringent goal for older people with history of severe hypoglycemia, advanced complications or extensive comorbid conditions $(n=27)$. Moreover, area under receiver operating characteristic curve (AUC) was used to assess the discrimination of CDMSS for medication adherence (binary VAS; $<$ vs $\geq 80$ ) and better glycemic control (binary HbA1c; $<$ vs $\geq$ individualized optimal treatment targets). Analyses were performed with Stata software, version 14.0 (Stata Corp, College Station, TX, USA). Two-sided $P$-values $<0.05$ were considered to be statistically significant.

\section{Results}

A total of $210(81.7 \%)$ patients were recruited from outpatient clinics, and 47 (18.3\%) patients were recruited from inpatient wards. The characteristics of patients are presented in Table 1. The mean age and BMI of this sample were 56.95 (SD: 11.22 ) years and 24.55 (SD: 4.37 ) $\mathrm{kg} / \mathrm{m}^{2}$, and $44.4 \%$ were females. The mean T2D duration was 7.94 (SD: 6.40) years and patients have been taking diabetes medications for an average of 6.91 (SD: 6.21) years. Patients were on an average of 2.28 (SD: 1.28) diabetes medications, and the daily frequency of medication intake was 2.85 (SD: 1.55) times. Moreover, the mean levels of FPG and HbA1c were 8.53 (SD: 2.73 ) $\mathrm{mmol} / \mathrm{L}$ and 8.70 (SD: 2.30 ) \%, respectively. In addition, the score for the CDMSS was 42.86 (SD: 9.32), and it was 78.48 (SD: 19.95) for the VAS and 2.99 (SD: 0.93 ) for the CDDS-15, respectively.

The detailed response to the CDMSS is shown in Table 2. Patients were more likely to report no confidence under following circumstances: 1) when the medications cause some side effects (item $3 ; 20.6 \%$ ); 2) when the medications cost a lot of money (item 5, 19.8\%); 3) when the medications sometimes make the patients tired (item 13, 20.6\%); 4) when the patients are confused (item 17, 25.7\%) and 5) when the patients' vision is blurry (item 18, 22.2\%).

The exploratory factor analysis of the CDMSS using quartimax rotation yielded three factors, and the results are shown in Table 3. In addition, the results were similar when using the oblique rotation method after adjusting for possible correlations among factors. The Kaiser-Meyer-Olkin measure of sampling adequacy was 0.94, and the Bartlett's test of sphericity yielded a significant value $\left(\chi^{2}=3,732.03\right.$;
Table I General characteristics of patients $(n=257)$

\begin{tabular}{|c|c|}
\hline Characteristics & n (\%) or mean \pm SD \\
\hline \multicolumn{2}{|l|}{ Sex } \\
\hline Men & $143(55.6 \%)$ \\
\hline Women & 114 (44.4\%) \\
\hline Age, years & $57.0 \pm 11.2$ \\
\hline Body mass index, $\mathrm{kg} / \mathrm{m}^{2}$ & $24.6 \pm 4.37$ \\
\hline \multicolumn{2}{|l|}{ Education levels } \\
\hline Primary school & 45 (I7.5\%) \\
\hline Secondary school & $91(35.4 \%)$ \\
\hline High school and above & $121(47.1 \%)$ \\
\hline \multicolumn{2}{|l|}{ Marriage status } \\
\hline Married & $235(91.4 \%)$ \\
\hline Separated/divorced & II (4.28\%) \\
\hline Widowed & $7(2.72 \%)$ \\
\hline Never married & $4(1.56 \%)$ \\
\hline \multicolumn{2}{|l|}{ Employment status } \\
\hline Unemployed & 44 (I7.I\%) \\
\hline Employed & $95(37.0 \%)$ \\
\hline Retired & $118(45.9 \%)$ \\
\hline \multicolumn{2}{|l|}{ Monthly salary (RMB) } \\
\hline$\leq 2,000$ & $54(21.0 \%)$ \\
\hline $2,00 I-4,000$ & $114(44.4 \%)$ \\
\hline $4,001-8,000$ & $67(26.1 \%)$ \\
\hline$>8,000$ & $22(8.56 \%)$ \\
\hline \multicolumn{2}{|l|}{ Monthly medical expenditure (RMB) } \\
\hline$\leq 500$ & $132(51.4 \%)$ \\
\hline $50 I-1,000$ & $93(36.2 \%)$ \\
\hline $\mathrm{I}, 00 \mathrm{I}-2,000$ & $27(10.5 \%)$ \\
\hline$>2,000$ & $5(1.94 \%)$ \\
\hline History of T2D & $96(37.4 \%)$ \\
\hline Duration since T2D diagnosis & $7.94 \pm 6.40$ \\
\hline Fasting plasma glucose, $\mathrm{mmol} / \mathrm{L}$ & $8.53 \pm 2.73$ \\
\hline $\mathrm{HbAlc} \%$ & $8.70 \pm 2.30$ \\
\hline Having T2D complications & $163(63.4 \%)$ \\
\hline Having other diseases & $108(42.0 \%)$ \\
\hline Duration of T2D medication usage & $6.91 \pm 6.21$ \\
\hline \multicolumn{2}{|l|}{ T2D medication types ${ }^{\mathrm{a}}$} \\
\hline Oral medications & $234(91.1 \%)$ \\
\hline Insulin therapy & $87(33.8 \%)$ \\
\hline Others & $8(3.11 \%)$ \\
\hline Number of T2D medications usage & $2.28 \pm 1.28$ \\
\hline Daily frequency of T2D medications & $2.85 \pm 1.55$ \\
\hline Concurrent usage of other medications & $116(45.1 \%)$ \\
\hline Encountered medication side effects & $76(29.6 \%)$ \\
\hline Perception of T2D & $6.26 \pm 2.97$ \\
\hline Perception of T2D medications & $6.04 \pm 3.06$ \\
\hline Perception of T2D medication efficacy & $6.74 \pm 2.44$ \\
\hline Score of CDMSS & $42.9 \pm 9.32$ \\
\hline Score of VAS & $78.5 \pm 20.0$ \\
\hline Score of CDDS-I5 & $2.99 \pm 0.93$ \\
\hline
\end{tabular}

Note: a68 patients used both oral medications and insulin therapy.

Abbreviations: CDDS-15, 15-item Chinese version of Diabetes Distress Scale; CDMSS, Chinese version of Diabetes Medication Self-efficacy Scale; HbAlc, hemoglobin Alc; RMB, Ren Min Bi (Chinese currency); T2D, type 2 diabetes; VAS, Visual Analog Scale.

$d f=171 ; P<0.001)$. Among the 19 items, a total of 8 items loaded on two factors: item 7 (you do not have any symptoms of diabetes), item 8 (you are with family members), item 10 (you feel you do not need them), item 15 (you feel okay), item 
Table 2 Patient response to the Diabetes Medication Self-efficacy Scale ( $n=257)$

\begin{tabular}{|c|c|c|c|}
\hline $\begin{array}{l}\text { How sure are you that you can take your diabetes } \\
\text { medicines if ... }\end{array}$ & $\begin{array}{l}\text { Not at all sure, } \\
\text { n (\%) }\end{array}$ & $\begin{array}{l}\text { Somewhat sure, } \\
\text { n (\%) }\end{array}$ & $\begin{array}{l}\text { Very sure, } \\
\text { n (\%) }\end{array}$ \\
\hline I. You are busy at home & $22(8.6)$ & $126(49.0)$ & $109(42.4)$ \\
\hline 2. There is no one to remind you & $18(7.0)$ & $117(45.5)$ & $122(47.5)$ \\
\hline 3. They cause some side effects & $53(20.6)$ & $134(52.1)$ & $70(27.2)$ \\
\hline 4. You worry about taking them for the rest of your life & $45(17.5)$ & $|3|(5 \mid .0)$ & $81(31.5)$ \\
\hline 5. They cost a lot of money & $51(19.8)$ & $126(49.0)$ & $80(31.1)$ \\
\hline 6. You come home late from work or other activities & $34(13.2)$ & $136(52.9)$ & $87(33.9)$ \\
\hline 7. You do not have any symptoms of diabetes & $19(7.4)$ & $91(35.4)$ & $147(57.2)$ \\
\hline 8. You are with family members & $5(1.9)$ & $106(4 \mid .2)$ & $146(56.8)$ \\
\hline 9. You are in a public place & $24(9.3)$ & $122(47.5)$ & III (43.2) \\
\hline 10. You feel you do not need them & $44(17.1)$ & $95(37.0)$ & II 8 (45.9) \\
\hline II. You are traveling & $23(8.9)$ & $|2|(47.1)$ & II $3(44.0)$ \\
\hline 12. You take them more than once a day & $30(11.7)$ & $128(49.8)$ & $99(38.5)$ \\
\hline 13. They sometimes make you tired & $53(20.6)$ & $126(49.0)$ & $78(30.4)$ \\
\hline 14. You have other medicines to take & $35(13.6)$ & $140(54.5)$ & $82(31.9)$ \\
\hline 15. You feel okay & II (4.3) & $92(35.8)$ & I54 (59.9) \\
\hline 16. You are shaky or jittery & $48(18.7)$ & $120(46.7)$ & $89(34.6)$ \\
\hline I7. You are confused & $66(25.7)$ & I I 4 (44.4) & $77(30.0)$ \\
\hline 18. Your vision is blurry & $57(22.2)$ & I I 4 (44.4) & $86(33.5)$ \\
\hline 19. You have a headache & 48 (18.7) & $122(47.5)$ & 87 (33.9) \\
\hline
\end{tabular}

Note: Copyright @2016. Dove Medical Press. Reproduced from Sleath B, Carpenter DM, Blalock SJ, et al. Development of a new diabetes medication self-efficacy scale and its association with both reported problems in using diabetes medications and self-reported adherence. Patient Prefer Adherence. 2016;10:1003-1010.13

16 (you are shaky or jittery), item 17 (you are confused), item 18 (your vision is blurry) and item 19 (you have a headache). After excluding these items, the remaining 11 items loaded on only one factor. The single factor model accounted for

Table 3 Factor structure of Chinese version of Diabetes Medication Self-efficacy Scale $(n=257)$

\begin{tabular}{|c|c|c|c|}
\hline & Factor I & Factor 2 & Factor 3 \\
\hline CDMSS Ia & 0.79 & -0.29 & 0.05 \\
\hline CDMSS $2^{\mathrm{a}}$ & 0.77 & -0.25 & 0.01 \\
\hline CDMSS $3^{\mathrm{a}}$ & 0.72 & -0.08 & -0.34 \\
\hline CDMSS $4^{a}$ & 0.80 & -0.06 & -0.29 \\
\hline CDMSS $5^{\mathrm{a}}$ & 0.80 & -0.01 & -0.30 \\
\hline CDMSS $6^{a}$ & 0.85 & -0.05 & 0.01 \\
\hline CDMSS $7^{b}$ & 0.64 & -0.15 & 0.55 \\
\hline CDMSS $8^{\mathrm{b}}$ & 0.70 & -0.04 & 0.44 \\
\hline CDMSS 9a & 0.78 & 0.004 & 0.22 \\
\hline CDMSS $10^{b}$ & 0.52 & -0.02 & 0.41 \\
\hline CDMSS IIa & 0.72 & 0.07 & 0.33 \\
\hline CDMSS I $2^{\mathrm{a}}$ & 0.83 & 0.06 & 0.08 \\
\hline CDMSS I $3^{a}$ & 0.84 & 0.12 & -0.13 \\
\hline CDMSS I $4^{a}$ & 0.82 & 0.16 & -0.03 \\
\hline CDMSS $15^{b}$ & 0.55 & 0.02 & 0.56 \\
\hline CDMSS $16^{c}$ & 0.62 & 0.63 & 0.04 \\
\hline CDMSS I $17^{c}$ & 0.72 & 0.48 & -0.05 \\
\hline CDMSS I $8^{c}$ & 0.66 & 0.58 & -0.07 \\
\hline CDMSS $19^{\circ}$ & 0.70 & 0.54 & -0.06 \\
\hline
\end{tabular}

Notes: altems loaded on only one factor (Factor I). 'ltems loaded on two factors (Factor I and Factor 3). Items loaded on two factors (Factor I and Factor 2).

Abbreviation: CDMSS, Chinese version of Diabetes Medication Self-efficacy Scale.
$63.1 \%$ of the scale variance, with item loadings ranged from 0.72 to 0.85 . In addition, the 11 -item CDMSS (CDMSS-11) was strongly correlated with the 19-item CDMSS, and the Spearman's rank correlation coefficient was $0.96(P<0.001)$.

The Cronbach's $\alpha$ for internal consistency of the CDMSS-11 was 0.94 , and the ICC for test-retest reliability was 0.76 . The score of CDMSS-11 was positively correlated with that of VAS (Spearman's rank correlation coefficient $r=0.56, P<0.001)$, and it was negatively correlated with the score of CDDS-15, as well as blood levels of FPG and HbA1c ( $r=-0.38,-0.27$ and $-0.30 ; P<0.001$; Table 4). Subsequently, we evaluated the odds ratio (OR) (95\% CI) of the CDMSS-11 for adequate glycemic control (indicated by binary $\mathrm{HbA} 1 \mathrm{c}$ levels; $<$ vs $\geq$ individualized optimal treatment targets). In the first model after adjustment of age, sex, BMI and duration since T2D diagnosis, the OR was 0.90 (95\% CI 0.86-0.95; data not shown). The association remained the same in the second model after further adjustment of more variables

Table 4 The Spearman's rank correlation coefficients between the score of CDMSS-I I, VAS, CDDS-I 5 and blood levels of FPG and $\mathrm{HbAlc}$

\begin{tabular}{l|l|l}
\hline CDMSS-II & Spearman's $\boldsymbol{r}$ & $\boldsymbol{P}$-value \\
\hline VAS & 0.56 & $<0.00 \mathrm{I}$ \\
CDDS-I5 & -0.38 & $<0.00 \mathrm{I}$ \\
FPG & -0.27 & $<0.00 \mathrm{I}$ \\
HbAIc & -0.30 & $<0.00 \mathrm{I}$ \\
\hline
\end{tabular}

Abbreviations: CDDS-15, 15-item of Chinese version of Diabetes Distress Scale; CDMSS-I I, II-item Chinese version of Diabetes Medication Self-efficacy Scale; FPG, fasting plasma glucose; HbAIc, hemoglobin Alc; VAS, Visual Analog Scale. 
including demographic characteristics, medical history, medication usage (types, frequencies and side effects) and perception on T2D and related medications, and the OR was 0.90 (95\% CI 0.85-0.97; data not shown).

The discrimination of the CDMSS-11 for medication adherence and adequate glycemic control was further explored. Using the binary VAS ( $<$ vs $\geq 80$ ) as an indicator of good medication adherence, the AUC was 0.79 (95\% CI: 0.73-0.84; $P<0.001)$. The best cutoff value for the CDMSS-11 was 23.5 using Youden index in the receiver operating characteristic analysis, and the associated sensitivity and specificity were 0.77 and 0.70 , respectively. In addition, the AUC of CDMSS-11 for the binary HbAlc ( $<$ vs $\geq$ individualized optimal treatment targets) was 0.65 (95\% CI: 0.57-0.72; $P<0.001)$. The associated best cutoff value for the CDMSS-11 was 27.5 , with a sensitivity of 0.75 and a specificity of 0.49 .

Subsequently, we explored factors (demographic, clinical, medication usage and perception) that may influence diabetes medication self-efficacy, and thus assessed the relations between those factors and CDMSS-11. In the univariate analysis, 7 out of 23 factors were significantly associated with diabetes medication self-efficacy, including having T2D complications, under insulin therapy, concurrent usage of other medications, having encountered medication side effects, patient perception of T2D, T2D medications, and T2D medication efficacy (Table 5). In the multivariate analysis of all the continuous variables, perception of T2D medication efficacy remained its significant association with diabetes medication self-efficacy, while perception of T2D lost its statistical significance (Table 5).

\section{Discussion}

To the best of our knowledge, this was the first study to develop CDMSS-11 and evaluate its psychometric properties in a sample of Chinese T2D patients. The CDMSS-11 showed good validity and high test-retest reliability, and had moderate discrimination for identifying proper medication adherence (measured by VAS) and glycemic control (indicated by $\mathrm{HbA1c}$ ) in this Chinese population. In addition, the CDMSS-11 had significant correlation with

Table 5 Relations between factors (demographic, clinical, medication usage and perception) and diabetes medication self-efficacy measured by the II-item Chinese version of Diabetes Medication Self-efficacy Scale

\begin{tabular}{|c|c|c|c|}
\hline \multirow[t]{2}{*}{ Characteristics } & \multicolumn{2}{|l|}{ Univariate analysis } & \multirow{2}{*}{$\begin{array}{l}\text { Multivariate analysis } \\
\text { Beta-coefficient }(P \text {-value })\end{array}$} \\
\hline & Kruskal-Wallis $H\left(P\right.$-value $\left.{ }^{a}\right)$ & Spearman's $r\left(P\right.$-value $\left.{ }^{b}\right)$ & \\
\hline Sex & $\mathrm{I} .05(0.3 \mathrm{I})$ & & \\
\hline Age, years & & $-0.11(0.07)$ & $-0.10(0.13)$ \\
\hline Body mass index, $\mathrm{kg} / \mathrm{m}^{2}$ & & $0.06(0.35)$ & $0.06(0.27)$ \\
\hline Education levels & $5.44(0.07)$ & & \\
\hline Marriage status & $\mathrm{I} .0 \mathrm{I}(0.80)$ & & \\
\hline Employment status & $2.75(0.25)$ & & \\
\hline Monthly salary & $7.33(0.06)$ & & \\
\hline Monthly medical expenditure & $0.28(0.96)$ & & \\
\hline History of T2D & $0.01(0.91)$ & & \\
\hline Duration since T2D diagnosis & & $-0.09(0.13)$ & $-0.24(0.08)$ \\
\hline Having T2D complications & $7.69^{d}(0.01)$ & & \\
\hline Having other diseases & $\mathrm{I} .47(0.23)$ & & \\
\hline Duration of T2D medication usage & & $-0.04(0.53)$ & $0.21(0.13)$ \\
\hline \multicolumn{4}{|l|}{ T2D medication types } \\
\hline Under oral medications & $2.26(0.13)$ & & \\
\hline Under insulin therapy & $5.44^{e}(0.02)$ & & \\
\hline Under other medications & $2.90(0.09)$ & & \\
\hline Number of T2D medications usage & & $-0.01(0.92)$ & $0.01(0.94)$ \\
\hline Daily frequency of T2D medications & & $-0.08(0.20)$ & $-0.08(0.32)$ \\
\hline Concurrent usage of other medications & $\mid 2.8 \mathrm{I}^{\mathrm{e}}(0.05)$ & & \\
\hline Encountered medication side effects & $18.04^{f}(<0.001)$ & & \\
\hline Perception of T2D & & $0.28^{f}(<0.00 \mathrm{I})$ & $-0.01(0.92)$ \\
\hline Perception of T2D medications & & $0.33^{f}(<0.001)$ & $0.25(0.05)$ \\
\hline Perception of T2D medication efficacy & & $0.3 \mathrm{I}^{\mathrm{f}}(<0.00 \mathrm{I})$ & $0.18^{\mathrm{e}}(0.012)$ \\
\hline
\end{tabular}

Notes: ${ }^{a} P$-values were calculated based on the Kruskal-Wallis $H$ test for categorical variables; ${ }^{b} P$-values were calculated based on the $S$ pearman's rank correlation coefficient for continuous variables in the univariate analysis; ${ }^{c} P$-values were calculated based on the linear regression for continuous variables in the multivariate analysis; ${ }^{d} P<0.0 \mathrm{I}$; ${ }_{\mathrm{e}} \mathrm{P}<0.05$; ${ }^{\mathrm{f}} \mathrm{P}<0.001$.

Abbreviation: T2D, type 2 diabetes. 
medication adherence (measured by the VAS), diabetes distress (measured by the CDDS-15) and glycemic control (indicated by FPG and HbA1c levels). Furthermore, several factors that may have significant impact on medication selfefficacy were identified.

Exploratory factor analysis is a useful statistical tool to explore the structure of a construct and reveals the number of factors that may explain the interrelations among a set of items. ${ }^{23}$ The DMSS was developed among a sample $(n=51)$ of mostly African-Americans, ${ }^{13}$ and did not explore the structure of the questionnaire. The current study included more patients $(n=257)$, and explored the structure of CDMSS by the factor analysis. As a result, we have deleted eight double-loading items, and confirmed CDMSS as a single-factor structure with 11 items. In addition to the statistical analysis, we also looked at the culture context for the deletion of these items. In the current Chinese population, very few patients reported to have no symptoms of diabetes, or felt ok/do not need the medications, therefore, items 7, 10 and 15 were less relevant to this population and thus were deleted. In addition, items 16-19 were redundant because most patients felt that these items were unrelated to the medication intake. The 11-item CDMSS not only had very high correlation with the 19-item CDMSS (Spearman's rank correlation coefficient $r=0.96$; $P<0.001$ ), but also had better internal consistency than the 19-item DMSS (Cronbach's $\alpha=0.94$ vs 0.86 ), ${ }^{13}$ as well as good test-retest reliability (ICC $=0.76$ ). However, further studies with larger number of participants in Chinese or other Asian populations were required to validate our findings of construct structure, validity and reliability. In addition, we have found a positive correlation between diabetes medication self-efficacy and medication adherence using the continuous VAS score as an indicator $(r=0.56 ; P<0.001)$. In consistent with our results, Sleath et $a{ }^{13}$ have also observed a positive relation between diabetes medication self-efficacy and medication adherence in the original development study (OR of DMSS was 1.17 ; 95\% CI, 1.05-1.30).

Moreover, the current study was the first to show that diabetes medication self-efficacy measured by the CDMSS-11 was negatively correlated with diabetes distress measured by the CDDS-15, and blood levels of FPG and HbAlc. Our results implicated that higher diabetes medication selfefficacy was associated with less diabetes distress and better glycemic control. In congruent with our findings, a US study using another diabetes self-efficacy scale and the original 17 -item DDS has also reported a negative correlation between diabetes self-efficacy and diabetes distress among 898 T2D patients,${ }^{24}$ and Ting et al,${ }^{21}$ who developed and validated
CDDS-15 among a Chinese population in Hong Kong, have reported a significant association between diabetes distress and glycemic indices. To test the robustness of the results, we have further dichotomized HbAlc as a binary outcome $(<\mathrm{vs} \geq$ individualized optimal treatment targets) and controlled for potential confounding factors. Consistently, we have observed an inverse association between diabetes medication self-efficacy (continuous) and glycemic control.

Furthermore, our study found that the CDMSS-11 has good discriminatory utility for the identification of medication adherence and moderate discrimination for the glycemic control, and the respective AUCs were 0.79 (95\% CI: $0.73-0.84)$ and 0.65 (95\% CI: 0.57-0.72). Thus, our findings suggested that CDMSS-11 may be a useful tool for clinicians to assess and predict problems related to T2D self-management, so that subsequent individualized interventions and treatments can be introduced. In addition, we have further identified some factors that were significantly correlated with diabetes medication self-efficacy, which provided additional information for clinicians to evaluate patient medication self-efficacy, and thus may add value to identification of medication adherence.

\section{Strengths and limitations}

The major strength of the current study was its multi-center design with big sample size. In addition, after we translated the instrument to Chinese, we conducted pilot study to make sure the translated version adapted to Chinese culture and was easy to understand. Moreover, we have used comprehensive statistical analyses to assess the psychometric properties of CDMSS-11. However, some limitations merit consideration. First of all, the current study was cross-sectional design and thus cannot elucidate prospective association between medication self-efficacy and medication adherence. Thus, we planned to follow these patients up to evaluate their subsequent T2D self-management status. In addition, the VAS for measuring medication adherence was a self-reported tool, and thus may have resulted in response bias from patients. Furthermore, the three cities selected for the current study were all coastal areas with developed economy in China, and thus may not represent the less-developed cities, therefore, our results may not be generalizable to those cities and additional studies are needed to validate our results.

\section{Conclusion}

The current study supported the CDMSS- 11 as a valid and reliable instrument to measure medication self-efficacy among Chinese T2D patients. The CDMSS-11 may also 
be a useful tool in clinical practice to identify problems of medication adherence and glycemic controls. Further studies are needed to validate our findings and explore the underlying mechanisms.

\section{Ethical approval}

All procedures performed in studies involving human participants were in accordance with the ethical standards of the institutional research committee and with the 1964 Declaration of Helsinki and its later amendments or comparable ethical standards.

\section{Informed consent}

Informed consent was obtained from all individual participants included in the study.

\section{Data availability statement}

The data that support the findings of this study are available on reasonable request from the corresponding author $\mathrm{MZ}$. The data are not publicly available due to their containing information that could compromise the privacy of research participants.

\section{Acknowledgments}

We thank all the doctors and nurses from the three hospitals who involved in participant recruitment and data collection, especially Dr Ronghua Song, Professor Liang Tang, Dr Qiaofeng Yan and Dr Qini Shi. We also thank Professor Mingmei Wu from Xiamen City University, Professor Yifeng Lu from China Pharmaceutical University and Dr Hongmei Li from Fudan University for the contribution to the CDMSS translation. In addition, we thank Professor Betsy Sleath from Eshelman School of Pharmacy, University of North Carolina at Chapel Hill, for granting us to use DMSS and translate into Chinese. The research did not receive any specific grant from funding agencies in the public, commercial or not-for-profit sectors. Fudan University funded the current study.

\section{Author contributions}

$\mathrm{MZ}$ and XL conceived the study, interpreted the data, and critically revised the reports. JZ designed the study, translated the scale, recruited participants, collected data, performed statistical analysis, drafted and critically revised the reports. YW translated the scale, performed statistical analysis, drafted and critically revised the reports. XY, LX and JY recruited participants, collected data and critically revised the reports. All authors revised and approved the final report. All authors contributed toward data analysis, drafting and revising the paper and agree to be accountable for all aspects of the work.

\section{Disclosure}

The authors report no conflicts of interest in this work.

\section{References}

1. International Diabetes Federation. IDF Diabetes Atlas. 8th ed. 2017. Available from:https://www.idf.org/e-library/epidemiology-research/ diabetes-atlas.html. Accessed October 08, 2018.

2. Evans JM, Newton RW, Ruta DA, Macdonald TM, Morris AD. Socioeconomic status, obesity and prevalence of Type 1 and Type 2 diabetes mellitus. Diabet Med. 2000;17(6):478-480.

3. Bruno G, Runzo C, Cavallo-Perin P, et al; Piedmont Study Group for Diabetes Epidemiology. Incidence of type 1 and type 2 diabetes in adults aged 30-49 years: the population-based registry in the province of Turin, Italy. Diabetes Care. 2005;28(11):2613-2619.

4. Holman N, Young B, Gadsby R. Current prevalence of Type 1 and Type 2 diabetes in adults and children in the UK. Diabet Med. 2015;32(9): $1119-1120$

5. Xu Y, Wang L, He J, et al; 2010 China Noncommunicable Disease Surveillance Group. Prevalence and control of diabetes in Chinese adults. JAMA. 2013;310(9):948-959.

6. Egede LE, Gebregziabher M, Echols C, Lynch CP. Longitudinal effects of medication nonadherence on glycemic control. Ann Pharmacother. 2014;48(5):562-570.

7. Krass I, Schieback P, Dhippayom T. Adherence to diabetes medication: a systematic review. Diabet Med. 2015;32(6):725-737.

8. Zhou Y, Liao L, Sun M, He G. Self-care practices of Chinese individuals with diabetes. Exp Ther Med. 2013;5(4):1137-1142.

9. Bandura A. Self-Efficacy: The Exercise of Control. New York, NY: W H Freeman/Times Books/Henry Holt \& Co; 1997.

10. Gherman A, Schnur J, Montgomery G, et al. How are adherent people more likely to think? A meta-analysis of health beliefs and diabetes self-care. Diabetes Educ. 2011;37(3):392-408.

11. Wu SF, Lee MC, Liang SY, et al. Effectiveness of a self-efficacy program for persons with diabetes: a randomized controlled trial. Nurs Health Sci. 2011;13(3):335-343.

12. Nakahara R, Yoshiuchi K, Kumano H, et al. Prospective study on influence of psychosocial factors on glycemic control in Japanese patients with type 2 diabetes. Psychosomatics. 2006;47(3):240-246.

13. Sleath B, Carpenter DM, Blalock SJ, et al. Development of a new diabetes medication self-efficacy scale and its association with both reported problems in using diabetes medications and self-reported adherence. Patient Prefer Adherence. 2016;10:1003-1010.

14. American Diabetes Association. 2. Classification and Diagnosis of Diabetes. Diabetes Care. 2016;39(Suppl 1):S13-S22.

15. Brislin RW. Back-Translation for Cross-Cultural Research. J Cross Cult Psychol. 1970;1(3):185-216.

16. Ahmed N, Bestall JC, Payne SA, Noble B, Ahmedzai SH. The use of cognitive interviewing methodology in the design and testing of a screening tool for supportive and palliative care needs. Support Care Cancer. 2009;17(6):665-673.

17. Freyd M. The Graphic Rating Scale. J Educ Psychol. 1923;14(2): 83-102.

18. Wewers ME, Lowe NK. A critical review of visual analogue scales in the measurement of clinical phenomena. Res Nurs Health. 1990;13(4): 227-236.

19. Amico KR, Fisher WA, Cornman DH, et al. Visual analog scale of ART adherence: association with 3-day self-report and adherence barriers. J Acquir Immune Defic Syndr. 2006;42(4):455-459. 
20. Nau DP, Steinke DT, Williams LK, et al. Adherence analysis using visual analog scale versus claims-based estimation. Ann Pharmacother. 2007;41(11):1792-1797.

21. Ting RZ, Nan H, Yu MW, et al. Diabetes-related distress and physical and psychological health in chinese type 2 diabetic patients. Diabetes Care. 2011;34(5):1094-1096.

22. American Diabetes Association. 5. Glycemic Targets. Diabetes Care. 2016; 39(Suppl 1):S39-S46.
23. Pett MA, Lackey NR, Sullivan JJ. Making Sense of Factor Analysis: The Use of Factor Analysis for Instrument Development in Health Care Research. Thousand Oaks, CA: Sage; 2003.

24. Fisher L, Hessler DM, Polonsky WH, Mullan J. When is diabetes distress clinically meaningful?: establishing cut points for the Diabetes Distress Scale. Diabetes Care. 2012;35(2):259-264.

\section{Publish your work in this journal}

Patient Preference and Adherence is an international, peer-reviewed, open access journal that focuses on the growing importance of patient preference and adherence throughout the therapeutic continuum. Patient satisfaction, acceptability, quality of life, compliance, persistence and their role in developing new therapeutic modalities and compounds to optimize clinical outcomes for existing disease states are major areas of interest for the journal. This journal has been accepted for indexing on PubMed Central. The manuscript management system is completely online and includes a very quick and fair peer-review system, which is all easy to use. Visit http://www. dovepress.com/testimonials.php to read real quotes from published authors.

Submit your manuscript here: http://www.dovepress.com/patient-preference-and-adherence-journal 